\title{
Harvester : an edge service harvesting heterogeneous resources for ATLAS
}

\author{
Tadashi Maeno ${ }^{1 *}$, Fernando Harald Barreiro Megino², Doug Benjamin ${ }^{3}$, David Cameron ${ }^{4}$, \\ John Taylor Childers ${ }^{3}$, Kaushik $\mathrm{De}^{2}$, Alessandro De Salvo ${ }^{5}$,Andrej Filipcic ${ }^{6}$, John Hover ${ }^{1}$, \\ FaHui Lin $^{7}$, Danila Oleynik ${ }^{2}$, on behalf of the ATLAS Collaboration \\ ${ }^{1}$ Brookhaven National Laboratory, NY, USA \\ ${ }^{2}$ University of Texas at Arlington, TX, USA \\ ${ }^{3}$ Argonne National Laboratory, IL, USA \\ ${ }^{4}$ University of Oslo, Oslo, Norway \\ ${ }^{5}$ Sapienza Universita e INFN, Roma I, Roma, Italy \\ ${ }^{6}$ Jozef Stefan Institute, Ljubljana, Slovenia \\ ${ }^{7}$ Academia Sinica, Taipei, Taiwan
}

Abstract. The Production and Distributed Analysis (PanDA) system has been successfully used in the ATLAS experiment as a data-driven workload management system. The PanDA system has proven to be capable of operating at the Large Hadron Collider data processing scale over the last decade including the Run 1 and Run 2 data taking periods. PanDA was originally designed to be weakly coupled with the WLCG processing resources. Lately the system is revealing the difficulties to optimally integrate and exploit new resource types such as HPC and preemptible cloud resources with instant spin-up, and new workflows such as the event service, because their intrinsic nature and requirements are quite different from that of traditional grid resources. Therefore, a new component, Harvester, has been developed to mediate the control and information flow between PanDA and the resources, in order to enable more intelligent workload management and dynamic resource provisioning based on detailed knowledge of resource capabilities and their real-time state. Harvester has been designed around a modular structure to separate core functions and resource specific plugins, simplifying the operation with heterogeneous resources and providing a uniform monitoring view. This paper will give an overview of the Harvester architecture, current status with various resources, and future plans.

\section{Introduction}

The Production and Distributed Analysis (PanDA) system [1] has been developed to meet ATLAS [2] production and analysis requirements for a data-driven workload

${ }^{1 *}$ Corresponding author: tmaeno@bnl.gov 
management system capable of operating at LHC [3] data processing scale. PanDA scalability has been demonstrated in ATLAS through the rapid increase in usage over the last decade. PanDA was designed to have the flexibility to adapt to emerging computing technologies in processing, storage, networking and distributed computing middleware. The flexibility has been successfully demonstrated through the past years of evolving technologies adopted by computing centers in ATLAS which span many continents. PanDA performed very well during the LHC data taking. The system had been producing high volumes of Monte Carlo samples and making large-scale diverse computing resources available for individual analysis. The PanDA system used to rely on a server-pilot paradigm where the PanDA server centrally manages workload with various granularities, such as task, job, and event, while the pilot executes jobs on compute resources. This model has been working well for the grid with 250,000 jobs concurrently running because the pilot works as an abstract layer on grid resources which have similar requirements in terms of computing power, walltime limit, memory size, and so on, but not very well for opportunistic resources, especially for HPCs. Each HPC center has a different edge service and operational policy, leading to an over-stretched architecture of the pilot and incoherent implementation at different HPCs. In addition, too many manual interventions were required to effectively fill available CPU resources at all HPC centers. Although some HPC sites had seamlessly been integrated with the grid through ARC Control Tower (aCT) [4], it was tightly coupled with ARC Compute Element [5] which could not be deployed at all HPC centers, especially at large HPC centers in US.

A new component, Harvester, has been developed to address those issues since December 2016 with wide collaboration of resource and PanDA experts. Harvester is a resource-facing service between the PanDA server and collection of pilots. It is stateless with a modular design to work with different resource types and workflows. The main Harvester objectives are as follows:

- It should be a common machinery for pilot provisioning on all ATLAS computing resources.

- It should provide a commonality layer bringing coherence to HPC implementations.

- It should add a capability to dynamically optimize CPU allocation among various resource types to remove batch-level static partitioning.

- It should integrate the PanDA system and resources more tightly for new advanced workflows.

We will present in this paper a brief overview of the Harvester architecture, current status with various resources, and plans for the future.

\section{Overview of the Harvester architecture}

Figure 1 shows a schematic view of the Harvester architecture. Harvester is a stateless service with a local master database and a central slave database. The local database is used for real-time bookkeeping close to resources, and the central database is periodically synchronized with the local database to provide the resource information to the PanDA server. The PanDA server uses this information together with global overview of workload distribution in order to orchestrate behaviour of Harvester instances. Therefore, 
communication between Harvester and the PanDA server is bidirectional. Two types of database engines are supported, sqlite 3 and MariaDB. Each Harvester instance can be configured to choose a proper database as well as the number of threads, the number of processes, and the number of physical nodes, depending on available runtime environment. Multiple agents are asynchronously running in a Harvester instance to take actions based on transition of job status in the local database. Figure 2 shows how Harvester instances work in the PanDA system. For example, Harvester is supposed to run on edge nodes at HPC centers where CPU and memory usage are strictly limited, and sqlite 3 and only one process are used in resource-limited environment. It is also possible to run Harvester instances outside of the HPC network if HPC centers allow remote access to compute resources. For the grid and cloud resource-rich environment, it is possible to run MariaDB and multiple-processes on the dedicated physical nodes.

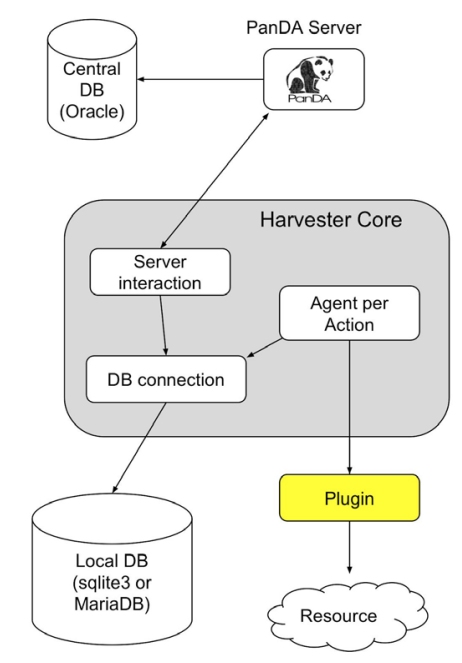

Fig. 1. Schematic view of Harvester architecture.

Harvester accesses resources through plugins which have been developed by resource experts. When Harvester instances run on edge nodes of HPC centers they access compute nodes through local HPC batch systems using HPC submission plugins. Input and output data are transferred with various plugins which use Rucio [6], Globus Online [7], gfal [8], and so on, according to data placement policy at each HPC center. When Harvester instances run outside of HPC network, different sets of plugins are used which access compute nodes through computing elements, SSH, and so on. For the grid, there is a Harvester pool on dedicated physical nodes which are centrally managed and access worker nodes through grid job submission engines like HTCondor [9] and aCT. The same Harvester pool can work for cloud with different sets of plugins which use GCE API [10], Kubernetes API [11], and so on to spin-up virtual machines or containers, or HTCondor to talk to virtual machines which are booted by other services. 


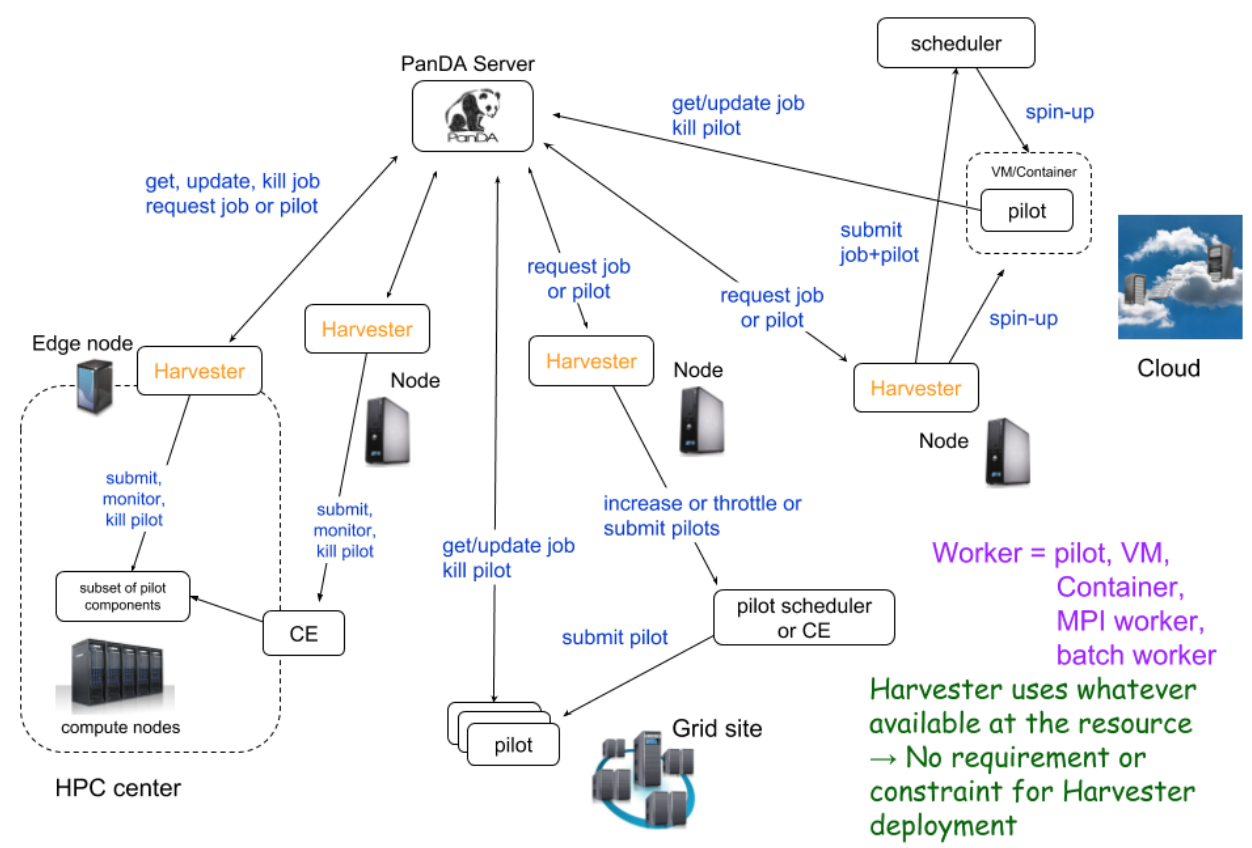

Fig. 2. Harvester instances in the PanDA system.

\section{Current status}

\subsection{Grid}

Migration to use Harvester for large scale production has been completed at CERN, Taiwan, Spanish and Italian sites, and migration at other sites are being scheduled. The runtime test framework for ATLAS offline software has been changed to use Harvester to cope with the intrinsic nature of intermittent workload submission. A mechanism has been developed to dynamically optimize resource partitioning based on current physics needs while getting rid of static batch-level partitioning, which is described in Ref [12]. Figure 3 shows that the mechanism managed to keep the ratio between the number of single core jobs and multi cores jobs at a site. 


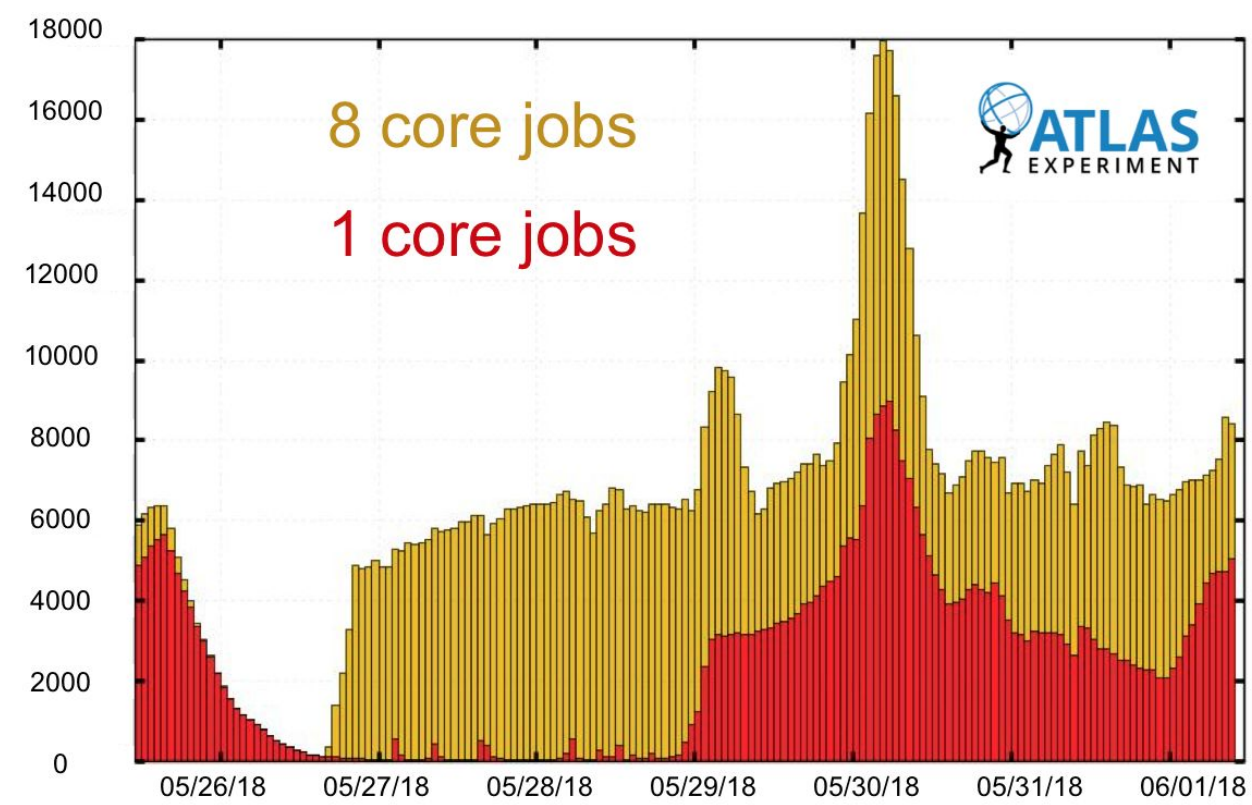

Fig. 3. The number of slots running single core jobs (in red) and multi core jobs (in yellow) at a site.

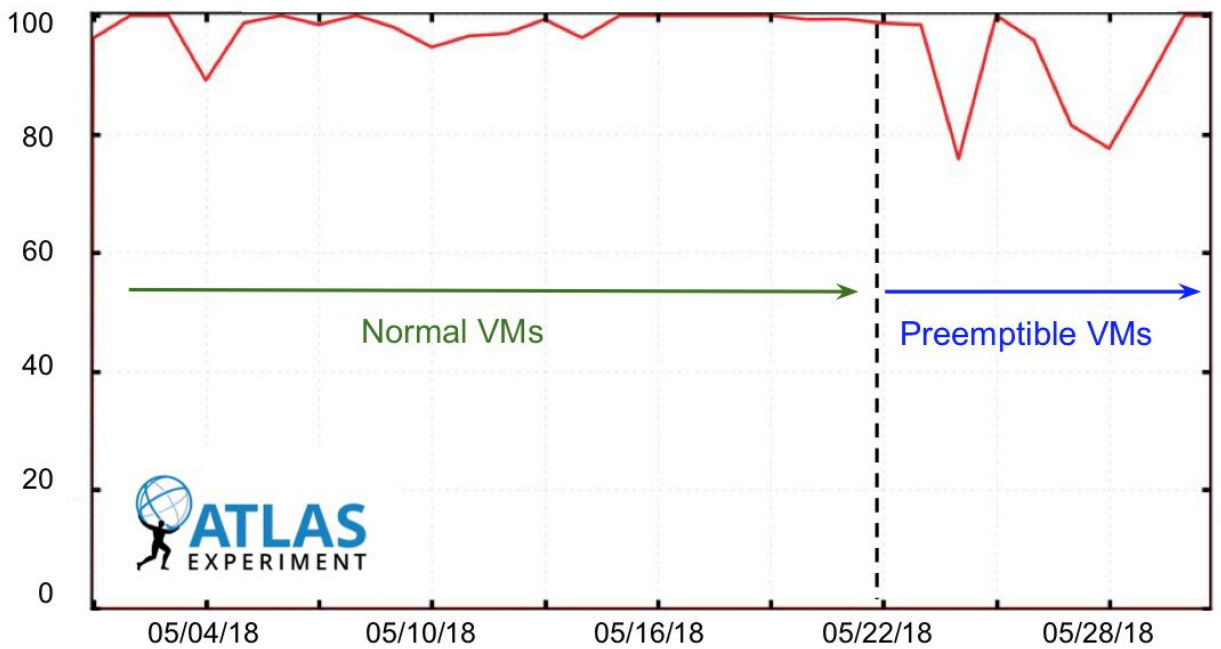

Fig. 4. Success rate of jobs running with GCE + Harvester.

\subsection{Cloud}

Cloud resources at CERN + Leibniz Supercomputing Centre + The University of Edinburgh with 1.2k CPU cores are running with Harvester in production, where virtual machines are booted by HTCondor. There are two major developments ongoing for cloud. 
The first development is for ATLAS High Level Trigger (HLT) CPU farm with 50k cores, aka Sim@P1, where resource availability widely fluctuates depending on needs for the original HLT usage [13]. Workload should be proactively allocated to the resource for rapid uptake even before the resource is available, while worker nodes should be released quickly as soon as HLT recovers the resource. The other development is to use native cloud API, such as GCE, EC2, and Kubernetes API. Plugins with GCE API have been successfully demonstrated in the context of the Data Ocean project [14] with GCE, Google Storage, and preemptible virtual machines. Figure 4 shows the success rate of jobs submitted with GCE and Harvester, where on 22 May 2018 the resource was reconfigured to use preemptible virtual machines instead of normal virtual machines to see the effect of switching. The success rate got worse because some jobs were terminated while they were still running.

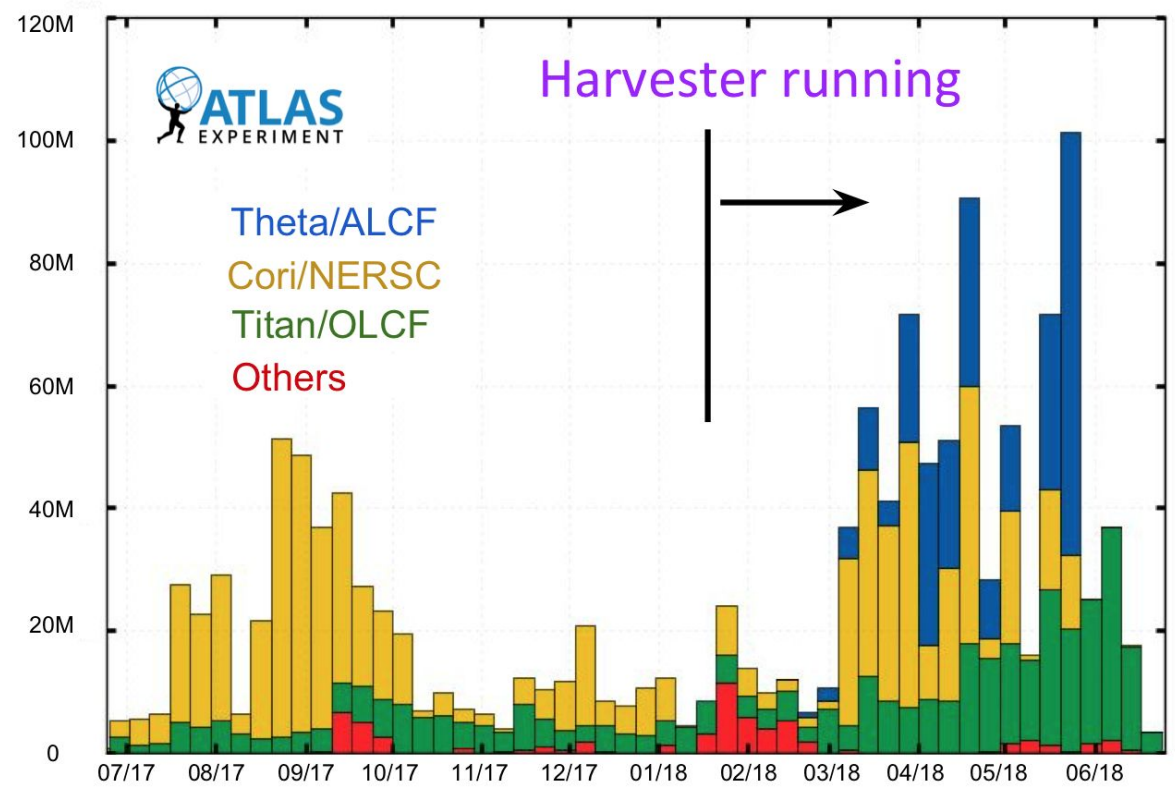

Fig. 5. The number of events processed per week at US HPCs for last 12 months.

\subsection{HPC}

Harvester has been running in production at Theta/ALCF [15], Titan/OLCF [16], Cori/NERSC [17] since February 2018 with a mechanism to dynamically combine many PanDA jobs to a single batch submission. Figure 5 shows the number of events processed per week at US HPCs for the last 12 months where there has been a steady increase since Harvester was up and running. The number of events at Cori/NERSC shown in yellow has not increased well since May 2018 because it had consumed all CPU allocation by then. Many development activities are going in parallel: Combination of jumbo payload and event service [18, 19] is going to address difficulties in payload sizing for HPCs. Operational policies at HPC centers drive the need for large payloads, while the system has to be protected against early termination due to preemption and/or inaccurate estimation of execution time. Some HPCs are being integrated to the grid infrastructure with HTCondor or ARC computing elements. The ability to dynamically change the payload size has been 
enabled to fill HPC with optimal payloads based on real-time HPC batch system information. There is also an idea to use a data streaming service and local cache service at HPC centers to dynamically deliver data to compute nodes on demand. This has to be implemented in line with ATLAS Event Streaming service developments [20].

\section{Beyond ATLAS}

Harvester is experiment agnostic. Six Harvester instances have been configured and ready to use for non-ATLAS experiment in BigPanDA project, including one regional instance at Thomas Jefferson Lab [21]. Various payloads have been tested, also with Next Generation Executer [22]. A new plugin has been developed so that Harvester can talk to other workload management systems than PanDA, which will expand Harvester usage more into other experiments.

\section{Future plans}

New developments and challenges are still coming. The entire ATLAS grid is migrating to Harvester for production as well as for analysis. The mechanism of dynamic resource partitioning will be enhanced to optimize resource allocation between production and analysis. There is a plan to develop a pilot-based information service to collect node-level real-time information on the grid, and the PanDA server and Harvester will schedule workload to optimize resource usage more efficiently by using this information instead of site-level or cluster-level static information. All HPCs are being integrated with the grid resources without any manual interventions. Finally, Harvester usage could be expanded beyond ATLAS.

\section{Conclusions}

Harvester has been developed since December 2016 with wide collaboration of resource and PanDA experts. Many development activities have been ongoing in parallel for various resources with coherent implementations to meet different requirements. Harvester is already in production for various resources, while there are still a lot of challenges to come.

\section{Acknowledgement}

This manuscript has been authored by employees of Brookhaven Science Associates, LLC under Contract No. DE-SC0012704 with the U.S. Department of Energy. The publisher by accepting the manuscript for publication acknowledges that the United States Government retains a nonexclusive, paid-up, irrevocable, worldwide license to publish or reproduce the 
published form of this manuscript, or allow others to do so, for United States Government purposes.

This research used resources of the National Energy Research Scientific Computing Center (NERSC), a U.S. Department of Energy Office of Science User Facility operated under Contract No. DE-AC02-05CH11231.

This research used resources of the Argonne Leadership Computing Facility, which is a DOE Office of Science User Facility supported under Contract DE-AC02-06CH11357.

This research used resources of the Oak Ridge Leadership Computing Facility at the Oak Ridge National Laboratory, which is supported by the Office of Science of the U.S. Department of Energy under Contract No. DE-AC05-00OR22725.

\section{References}

1. K. De et al., J. Phys. Conf. Ser. 664062035 (2015)

2. ATLAS Collaboration, J. Inst. 3 S08003 (2008)

3. Evans L and Bryant P (editors), J. Inst. 3 S08001 (2008)

4. J. K. Nilsen et al., J. Phys. Conf. Ser. 664062042 (2015)

5. ARC Compute Element [software], available from http://www.nordugrid.org/arc/ce/ [accessed 2018-11-22]

6. Rucio [software], available from https://rucio.cern.ch/ [accessed 2018-11-22]

7. Globus Online [software], available from https://www.globus.org/ [accessed 2018-11-22]

8. gfal [software] Available from https://dmc.web.cern.ch/projects/gfal-2/home [accessed 2018-11-22]

9. HTCondor [software], available from https://research.cs.wisc.edu/htcondor/ [accessed 2018-11-22]

10. GCE API [software], available from https://cloud.google.com/compute/docs/reference/rest/v1/ [accessed 2018-11-22]

11. Kubernetes API [software], available from ]https://kubernetes.io/docs/concepts/overview/kubernetes-api/ [accessed 2018-11-22]

12. F. H. Barreiro Megino et al., J. Phys. Conf. Ser. (to be published)

13. F. Berghaus et al., Phys. Conf. Ser. (to be published)

14. M. Lassnig et al., J. Phys. Conf. Ser. (to be published)

15. ALCF Theta, http://www.alcf.anl.gov/

16. OLCF Titan, https://www.olcf.ornl.gov/titan/

17. NERSC Cori, https://www.nersc.gov/

18. T. Wenaus et al., J. Phys. Conf. Ser. 664062065 (2015)

19. V. Tsulaia et al., J. Phys. Conf. Ser. 664092025 (2015)

20. N. Magini et al., J. Phys. Conf. Ser. (to be published)

21. P. Svirin et al. J. Phys. Conf. Ser. (to be published)

22. M. Turilli et al., arXiv preprint arXiv:1609.03484 (2016) 